\title{
Tangencies for Real and Complex Hénon Maps: An Analytic Method
}

\author{
John Erik Fornæss and Estela A. Gavosto
}

\section{CONTENTS}

1. Introduction

2. Formulas for the Parametrizations of the Unstable and Stable Manifold

3. Numerical Location of the Tangency

4. Proving the Existence of the Tangency

Acknowledgements

References
We present a method for computing generic homoclinic tangencies in the complex Hénon map, based on analytic parametrizations of the stable and unstable manifold, and we discuss applications and consequences of the existence of such tangencies.

\section{INTRODUCTION}

The Hénon map, first introduced in [Hénon 1976], is the diffeomorphism of the plane given by

$$
H_{a, b}(x, y)=\left(1-a x^{2}+b y, x\right) .
$$

Hénon observed experimentally that for $a=1.4$ and $b=0.3$ this map exhibits a strange attractor: there is a compact subset $K \subset \mathbb{R}^{2}$ such that given a point $\left(x_{0}, y_{0}\right)$ in an open set containing $K$, the sequence of iterates $H^{n}\left(x_{0}, y_{0}\right)$ converges to $K$, and the dynamics on $K$ has sensitive dependence on the initial condition.

Complex Hénon Maps are defined by

$$
\begin{aligned}
H_{a, b}: \mathbb{C}^{2} & \rightarrow \mathbb{C}^{2}, \\
(z, w) & \mapsto\left(1-a z^{2}+b w, z\right),
\end{aligned}
$$

where $a$ and $b$ are complex constants. They appear naturally as the first nontrivial generalization to two variables of the quadratic polynomial $P_{c}(z)=z^{2}+c$. It is well known from the classification in [Friedland and Milnor 1989] that Hénon maps are (up to conjugation) the degree-two polynomial authomorphisms of $\mathbb{C}^{2}$ with interesting dynamics. The complex Hénon mappings with real coefficients restricted to the real numbers give back the real Hénon maps. Thus many dynamical results for the complex map translate immediately to results for the real map.

Our work focuses on the study of a particular type of bifurcations: generic homoclinic tangencies. To 
define these bifurcations, let $P_{0}$ be a saddle fixed point and let $\lambda^{s}\left(P_{0}\right), \lambda^{u}\left(P_{0}\right)$ be the corresponding stable and unstable eigenvalues of $D H_{a, b}\left(P_{0}\right)$. The corresponding unstable manifold is

$$
M_{a, b}^{u}\left(P_{0}\right)=\left\{(z, w): H_{a, b}^{-n}(z, w) \rightarrow P_{0}\right\}
$$

and the stable manifold is

$$
M_{a, b}^{s}\left(P_{0}\right)=\left\{(z, w): H_{a, b}^{n}(z, w) \rightarrow P_{0}\right\} .
$$

For historical reasons, we will set $b=0.3$, but the ideas of our algorithm and method work for arbitrary complex $b$. Assume that locally $M_{a}^{u}\left(P_{0}\right)$ is $w=\Phi(a, z)$ and that $M_{a}^{s}\left(P_{0}\right)$ is $w=\Psi(a, z)$. Let $\sigma=\Phi-\Psi$. We say that $M_{a}^{u}$ and $M_{a}^{s}$ have a generic homoclinic tangency at $\left(a_{0}, z_{0}\right)$ if

$$
\begin{aligned}
\sigma\left(a_{0}, z_{0}\right) & =0, & \frac{\partial}{\partial z} \sigma\left(a_{0}, z_{0}\right) & =0 \\
\frac{\partial^{2}}{\partial z^{2}} \sigma\left(a_{0}, z_{0}\right) & \neq 0, & \frac{\partial}{\partial a} \sigma\left(a_{0}, z_{0}\right) & \neq 0 .
\end{aligned}
$$

The main importance of the existence of generic homoclinic tangencies arises from a remarkable result of Newhouse [1974] saying that, if a family of diffeomorphisms of $\mathbb{R}^{2}$ has a generic homoclinic tangency, there exists a parameter value $\alpha_{0}$ with a diffeomorphism with infinitely many attracting basins. Moreover (see [Robinson 1983]) there is an interval $I$ containing a dense subset $J$ with the corresponding diffeomorphisms having a generic homoclinic tangency. More recently, following the work in [Benedicks and Carleson 1991], Mora and Viana [1993] showed that the existence of generic homoclinic tangencies implies the existence of strange attractors.

In [Fornæss and Gavosto 1992], we proved the existence of a generic homoclinic tangency for

$$
\left(a_{0}, b_{0}\right)=(1.392 \ldots, 0.3) .
$$

This implies the existence of strange attractors for this value of the parameter. In addition, by [Newhouse 1974], the complex Hénon map has infinitely many attracting basins. These basins are particularly interesting since they are Fatou-Bieberbach domains, that is, biholomorphic copies of $\mathbb{C}^{2}$ that are proper subsets of $\mathbb{C}^{2}$. There are many open questions regarding the geometry of these domains. For example, how these infinitely many domains intertwine with each other. There are also many questions about the boundary of these domains. In particular, it has been shown in [Stens $\varnothing$ nes 1997] that there exist Fatou-Bieberbach domains with smooth boundary.

Another reason for the importance of generic homoclinic complex holomorphic tangencies is that, if a family $\Phi_{\alpha}$ of holomorphic mappings has a generic homoclinic tangency $\alpha_{0}$ at $Q$, then for each neighborhood $V$ of $Q$ there is an attracting periodic point in $V$ for some $\tilde{\alpha}_{0}$ close to $\alpha_{0}$. See [Gavosto $\geq 1999$ ]. Once the tangency is found, this same paper gives a constructive method to locate the basin of attraction, which is a Fatou-Bieberbach domain. Other situations where the importance of locating generic homoclinic tangencies is evident are described in [Buzzard 1997; Gavosto $\geq 1999$; Kan et al. 1995].

There are basically two methods to compute the unstable and stable manifolds of Hénon mappings. One is the method in [Kan et al. 1995], consisting in considering the forward iterates of a small curve through the fixed point.

Our approach, instead, consists in considering the analytic parametrization of these manifolds. The advantage of our method is that it allows us to use all the analytic tools in $\mathbb{C}$ and $\mathbb{C}^{2}$ to prove the necessary estimates to guarantee the existence of generic homoclinic tangencies. We have developed this approach in [Fornæss and Gavosto 1991; 1992], where we showed the existence of generic homoclinic tangencies for particular values of the parameter. These techniques have been also used in [Franceschini and Russo 1981] to show the existence of homoclinic intersections for the Hénon mapping. We wanted to continue searching for tangencies for different values of the parameters but the work in [Fornæss and Gavosto 1991; 1992] involves many hand-computed estimates. To avoid repeating those cumbersome computations, we wrote a program that checks those estimates for us.

We present here a rigorous general method to search for generic homoclinic tangencies. We also give some applications related to a conjecture in [Kan et al. 1995]. To simplify the exposition, we describe our method for mappings with real coefficients and look for tangencies in the real plane. A generic homoclinic tangency for the real Hénon 
map is also a complex generic homoclinic tangency for the complex Hénon map with real coefficients. The method presented can be easily adapted for the case of complex coefficients; we indicate the necessary modifications in the corresponding sections. The general algorithm involves the following steps:

A. Find inductive formulas for the analytic parametrizations of the unstable and stable manifolds.

B. Find upper bounds of the coefficients of the parametrizations found in Step A, for values of $a$ in a disk.

C. Using the upper bounds of Step B, find an optimal degree of truncation for the power series of the parametrizations.

D. Using these truncations, find a numerical approximation of the location of generic homoclinic tangencies in $a$ and the parameters of $M^{u}$ and $M^{s}$.

E. Check if the approximation of the location appears to be very close to tangency.

F. Verify the conditions of generic homoclinic tangency. If any of the conditions fails, go back to Step D or C according to the problem.

The numerical computations to find the first approximate location of the tangency are done using Pari. The estimates and the rest of the algorithm have been done using Mathematica and interval arithmetic with rational endpoints.

This paper is organized as follows. Section 2 describes steps A, B, and C. Section 3 discusses the numerical procedure of step $\mathrm{D}$ and the geometric method of E. Section 4 discusses the algorithms to check all the conditions of the generic homoclinic tangency. Finally, Section 5 gives some applications and further uses of the method.

Matthew Petro, a student of ours, carried out most of the programming of this method.

\section{FORMULAS FOR THE PARAMETRIZATIONS OF THE UNSTABLE AND STABLE MANIFOLD}

We will work with a range of parameter values where it is well known that the unstable and stable manifolds exist. In fact, one of the fixed points of the Hénon map is a saddle point. For example, for the values of the parameters, $a$ real in the interval $[0.4,1.5]$ and for $b=0.3$. We will consider mappings with real coefficients and we will search for tangencies in the real plane. From the functional equations (see [Fornæss and Gavosto 1992]) we find inductive formulas for the analytic parametrizations of the unstable and stable manifolds. The unstable manifold $M^{u}$ has a parametrization given by $g(a, \tau)=\left(X^{u}(\tau), Y^{u}(\tau)\right)$, and the stable manifold is given by $h(a, t)=\left(X^{s}(t), Y^{s}(t)\right)$, where

$$
\begin{aligned}
X^{u}(\tau, a) & =\sum_{n=0}^{\infty} U_{n}(a) \tau^{n} \\
& =X_{N}^{u}(\tau, a)+\sum_{n=N+1}^{\infty} U_{n}(a) \tau^{n} \\
Y^{u}(\tau, a) & =\sum_{n=0}^{\infty} U_{n}(a)\left(\tau / \lambda^{u}\right)^{n} \\
& =Y_{N}^{u}(\tau, a)+\sum_{n=N+1}^{\infty} U_{n}(a)\left(\tau / \lambda^{u}\right)^{n}, \\
X^{s}(t, a) & =\sum_{n=0}^{\infty} S_{n}(a)\left(t \lambda^{s}\right)^{n} \\
& =X_{M}^{s}(t, a)+\sum_{n=M+1}^{\infty} S_{n}(a)\left(t \lambda^{s}\right)^{n}, \\
Y^{s}(t, a) & =\sum_{n=0}^{\infty} S_{n}(a) t^{n} \\
& =Y_{M}^{s}(t, a)+\sum_{n=M+1}^{\infty} S_{n}(a) t^{n},
\end{aligned}
$$

where the eigenvalues $\lambda^{u}$ and $\lambda^{s}$ at the fixed point $\left(U_{0}(a), U_{0}(a)\right)$ are

$$
\begin{aligned}
& \lambda^{u}=-a U_{0}(a)-\sqrt{b+\left(a U_{0}(a)\right)^{2}} \\
& \lambda^{s}=-a U_{0}(a)+\sqrt{b+\left(a U_{0}(a)\right)^{2}}
\end{aligned}
$$

and the coefficients $U_{j}$ are given by

$$
U_{0}(a)=S_{0}(a)=\frac{b-1+\sqrt{(b-1)^{2}+4 a}}{2 a},
$$

$U_{1}(a)=S_{1}(a)=1$ (for normalization), and

$$
\begin{aligned}
& U_{n}(a)=-a \frac{\sum_{i=1}^{n-1} U_{i} U_{n-i}}{\left(\lambda^{u}\right)^{n}-b /\left(\lambda^{u}\right)^{n}+2 a U_{0}}, \\
& S_{n}(a)=-a \frac{\sum_{i=1}^{n-1} S_{i} S_{n-i}}{\left(\lambda^{s}\right)^{n}-b /\left(\lambda^{s}\right)^{n}+2 a S_{0}}
\end{aligned}
$$

for $n>1$.

The first necessary step to be able to control the truncation errors is to find formulas for the upper bounds of the coefficients. Following [Fornæss and 
Gavosto 1992] (which can be consulted for more details) we obtain explicit recurrence formulas for the upper bounds. To do this we use disc analysis. The upper bounds of $U_{n}(a)$ for $a$ in the disk $B\left(a_{0}, r\right)$ will be denoted by $V_{n}$; that is, given $a_{0}$ and $r>0$, we have $U_{n}\left(B\left(a_{0}, r\right)\right) \subset B\left(0, V_{n}\right)$.

We use the following notation. If $f$ is a function on $B\left(a_{0}, r\right)$, we denote by $\min f$ and $\max f$ the minimum and maximum of $|f(a)|$, for $a \in B\left(a_{0}, r\right)$. Let

$$
V_{0}:=\left|U_{n}\left(a_{0}\right)\right|+\max \left|\frac{\partial}{\partial a} U_{0}\right| r .
$$

To obtain the general formula for $V_{n}$, we define $K$ as the smallest integer such that

$$
\left|2 \max (a) \cdot V_{0}+\frac{b}{\left(\min \lambda^{u}\right)^{K}}\right|<\left|\lambda^{u}\left(a_{0}\right)\right|
$$

and we write

$$
U_{n}(a)=g_{n}(a) \sum_{i=1}^{n-1} U_{i}(a) U_{n-1}(a)
$$

with

$$
g_{n}(a):=-a\left(\left(\lambda^{u}\right)^{n}-\frac{b}{\left(\lambda^{u}\right)^{n}}+2 a U_{0}\right)^{-1}
$$

moreover we set

$$
\begin{aligned}
& D_{0}:=\max \left|\frac{\partial}{\partial a} U_{0}\right| \\
& D_{n}:=\left|\max \left(\frac{\partial}{\partial a} g_{n}\right)\right| \sum_{i=1}^{n-1} V_{i} V_{n-i} \\
& \quad+\left|\max g_{n}\right| \sum_{i=1}^{n-1}\left(D_{i} V_{n-i}+V_{i} D_{n-i}\right)
\end{aligned}
$$$$
\text { for } n \geq 1 \text {, }
$$

$A_{n}:=\left(\min \lambda^{u}\right)^{n}-\left|\lambda^{u}\left(a_{0}\right)\right| n \geq k$.

Then the general formula for $V_{n}$ is

$$
V_{n}:= \begin{cases}\left|U_{n}\left(a_{0}\right)\right|+D_{n} r & \text { if } n<K, \\ 1.001\left(\left|a_{0}\right|+r\right) \frac{\sum_{i=1}^{i=n-1} V_{i} V_{n-i}}{A_{n}} & \text { if } n \geq K .\end{cases}
$$

Now we can use these formulas for the upper bounds to find $N$ and to truncate the parametrizations of $M^{u}$. We write

$$
X^{u}(\tau, a)=\sum_{n=0}^{N} U_{n}(a) \tau^{n}+E(a, \tau, N) .
$$

To estimate $E(a, \tau, N)$, for $\tau \in\left[\tau_{\min }, \tau_{\max }\right]$, we use the $V_{n}$ 's, since

$$
|E(a, \tau, N)|=\left|\sum_{n=N+1}^{\infty} U_{n}(a) \tau^{n}\right| \leq \sum_{n=N+1}^{\infty} V_{n} \tau_{0}^{n}
$$

where $\tau_{0}=\max \left\{|\tau|: \tau \in\left[\tau_{\min }, \tau_{\max }\right]\right\}$. By analyticity, there exists a constant $s \in(0,1)$ and constants $C>0$ and $N_{0}>0$ such that $V_{N} \tau_{0}^{N} \leq C s^{N}$ for all $N>N_{0}$. Unless otherwise stated, $N$ will be assumed larger than $N_{0}$ for the remainder of the paper. The constant $s$ is chosen according to our experience with the rate of convergence of $U_{n}$. Similarly, we can obtain formulas for $Y^{u}, X^{s}$, and $Y^{s}$.

\section{NUMERICAL LOCATION OF THE TANGENCY}

Now that we have the parametrizations for both manifolds, we want to find an approximate location of generic homoclinic tangencies in $a$ and the parameters of $M^{u}$ and $M^{s}$. We consider the equations

$$
\left.\begin{array}{r}
X_{N}^{u}(\tau, a)-X_{M}^{s}(t, a)=0 \\
Y_{N}^{u}(\tau, a)-Y_{M}^{s}(t, a)=0 \\
T_{N, M}(a, \tau, t)=0,
\end{array}\right\}
$$

where

$$
T_{N, M}(a, \tau, t)=\frac{\partial X_{N}^{u}}{\partial \tau} \frac{\partial Y_{M}^{s}}{\partial t}-\frac{\partial X_{M}^{s}}{\partial t} \frac{\partial Y_{N}^{u}}{\partial \tau}
$$

and the indices $N, M$ indicate that we are considering the first $N$ or $M$ terms of the power series expansions. Geometrically, the first two equations impose the condition that the manifolds that approximate the unstable and stable manifold intersect. The third equation requires the two manifolds to be tangent. These equations will only look for a homoclinic tangency. We will verify later that the tangency is generic. We use Newton's method to find an approximate solution of the system $(3-1)$. Any other numerical method could be used at this step. We picked the basic Newton's method because it has a very simple algorithm, a desirable feature since we are working with high precision. A priori, we don't know if the system (3-1) has real solutions. In particular, if the tangency is not generic because the derivatives with respect to $a$ are zero, the determinant of the Jacobian is zero. Nevertheless, experimentally, we have observed that Newton's method is convergent for the right choice of the initial values. 
Newton's method gives us a number of iterates $k$ and $\left(a_{k}, \tau_{k}, t_{k}\right)$. The value of $a_{k}$ gives manifolds $M^{u}\left(a_{k}\right)$ and $M^{s}\left(a_{k}\right)$ and $\tau_{k}$ and $t_{k}$ give an approximate location of a possible homoclinic tangency. We also save the values corresponding to the previous iteration $k-1$ in Newton's method, $\left(a_{k-1}, \tau_{k-1}, t_{k-1}\right)$. Now, in the complex case, we check if $\left|a_{k}-a_{k-1}\right|$, $\left|\tau_{k}-\tau_{k-1}\right|$, and $\left|t_{k}-t_{k-1}\right|$ are very small. If not, we go back to Newton's method. In the real case, rather than just checking these distances, we have found it efficient to do the following. To find a close location to the tangency, we move the parameter $a$ to generate another unstable manifold, also very close to a homoclinic tangency, but on the other side on the unstable manifold $M^{s}$. The method is as follows.

First, we generate intervals in $\tau$ and $t$ of the form

$$
\begin{aligned}
& I_{k}^{\varepsilon_{1}}=\left[\tau_{k}-\varepsilon_{1}, \tau_{k}+\varepsilon_{1}\right], \\
& I_{k}^{\varepsilon_{2}}=\left[t_{k}-\varepsilon_{2}, t_{k}+\varepsilon_{2}\right],
\end{aligned}
$$

where $\varepsilon_{1}=\delta_{1}\left|\tau_{k}-\tau_{k-1}\right|, \varepsilon_{2}=\delta_{2}\left|t_{k}-t_{k-1}\right|$, and $\delta_{1}=\delta_{2}=2$. Next we check if the piece of the unstable manifold corresponding to $I_{k}^{\varepsilon_{1}}$ lies completely on one side of the unstable manifold. To do this, we have to check first that the range in $Y$-coordinate of the unstable manifold is contained in the range in the $Y$-coordinate of the stable manifold; that is, if $Y_{N}^{u}\left(I_{k}^{\varepsilon 1}\right) \subset Y_{M}^{s}\left(I_{k}^{\varepsilon_{2}}\right)$. If this is not true, we have to go back and consider a much bigger interval in $t$ (with a bigger $\delta_{2}$ ) and repeat the process. We are assuming that locally the unstable and stable manifolds are functions of $y$. Similarly, we could consider the case where the unstable and stable manifolds are functions of $x$.

Let $Q_{\tau}$ denote the point on the unstable manifold with parameter $\tau$. We consider three values of $\tau$ : the center and the two endpoints of the interval $I_{k}^{\varepsilon_{1}}$. We set $\tau_{k}^{1}=\tau_{k}-\varepsilon_{1}$ and $\tau_{k}^{2}=\tau_{k}+\varepsilon_{1}$. For each of these values of $\tau$, let $L\left(Q_{\tau}\right)$ denote the horizontal line through $Q_{\tau}$. Next we consider a line $l$ through $P=\left(X_{M}^{s}\left(a_{k}, t_{k}\right), Y_{M}^{s}\left(a_{k}, t_{k}\right)\right)$ with the same slope as the stable manifold. The intersection of $l$ and the line $L\left(Q_{\tau}\right)$ gives a point $I\left(Q_{\tau}\right)$ (see Figure 1 ). Next, we check the sign of the $x$-coordinates of $Q_{\tau}-I\left(Q_{\tau}\right)$, for $\tau=\tau_{k}^{1}, \tau_{k}^{2}$, and $\tau_{k}$. If they are not the same, the unstable manifold is not on one side of $M^{s}$. By continuity, we can keep on repeating this process with a smaller $\delta_{1}$ until the signs are the same.

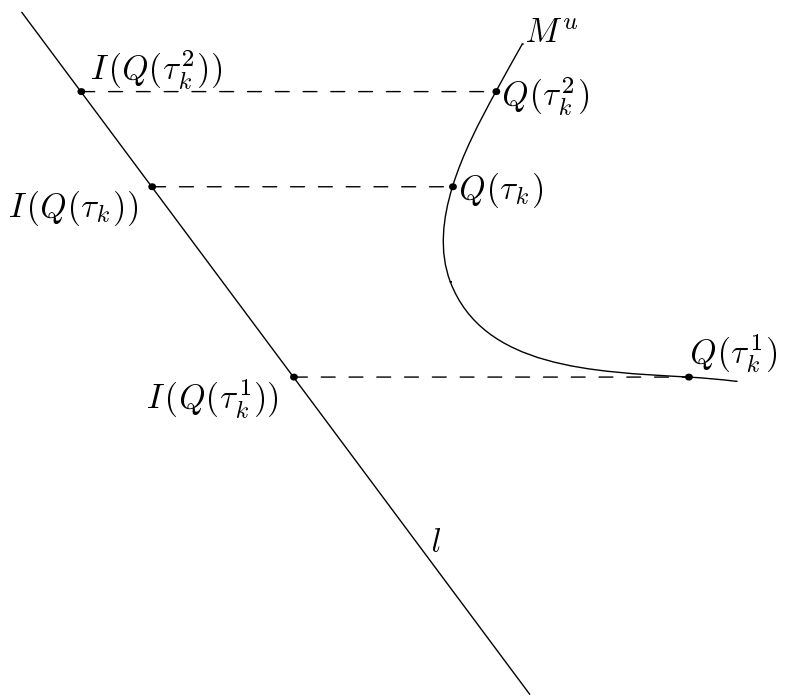

FIGURE 1. Definition of $I\left(Q_{\tau}\right)$.

The next step is to find an approximated value of how much $a$ has to be changed in order to move the unstable manifold to the other side of the stable manifold. For this, we calculate a first order approximation of the curve $C$, defined by following the points $Q_{\tau}=Q(\tau, a)$ in the unstable manifold as the parameter $a$ changes. The approximation of $C$ that we consider is given by

$r(a)=Q\left(\tau_{k}, a_{k}\right)+\frac{Q\left(\tau_{k}, a_{k}\right)-Q\left(\tau_{k-1}, a_{k-1}\right)}{a_{k}-a_{k-1}}\left(a-a_{k}\right)$.

We then compute the value of $a, a^{*}$, when this path intersects the stable manifold. After that we must test whether the points $Q\left(a^{*}, \tau_{k}^{1}\right)$ and $Q\left(a^{*}, \tau_{k}^{2}\right)$ move toward or away from the stable manifold; we do this by checking the difference of the $x$-coordinates between $Q\left(a^{*}, \tau_{k}^{1}\right)$ and $Q\left(a^{*}, \tau_{k}^{2}\right)$ and $Q\left(a_{k}, \tau_{k}^{1}\right)$ and $Q\left(a_{k}, \tau_{k}^{2}\right)$. Otherwise the interval $I_{k}^{\varepsilon_{1}}=\left[\tau_{k}^{1}, \tau_{k}^{2}\right]$ must be shortened again. After successfully completing this process, we define $a_{2}=2\left(a^{*}-a_{k}\right)+a_{k}$.

\section{PROVING THE EXISTENCE OF THE TANGENCY}

We must verify the conditions of generic homoclinic tangency. Recall that the unstable manifold is parametrized by $g(\tau)=\left(X^{u}(\tau), Y^{u}(\tau)\right)$ and the stable manifold by $h(t)=\left(X^{s}(t), Y^{s}(t)\right)$. If the derivatives $\partial Y^{u} / \partial \tau$ and $\partial Y^{s} / \partial t$ are not zero, we can describe the stable and unstable manifolds as graphs:

$$
x=\psi(a, y) \quad \text { and } \quad x=\varphi(a, y),
$$


respectively, for the stable and unstable manifolds. Equivalently, we can consider the manifolds as functions of $x$. We want to express the derivatives of $\varphi$ and $\psi$ in terms of derivatives of the power series expansions of the stable and unstable manifolds. We obtain these expressions using the implicit relationships

$$
\begin{aligned}
X^{u}(\tau, a) & =\varphi\left(a, Y^{u}(\tau, a)\right), \\
X^{s}(t, a) & =\psi\left(a, Y^{s}(t, a)\right),
\end{aligned}
$$

to derive the formulas for the derivatives.

Verifying the conditions of the existence of the generic homoclinic tangency involves the systematic study of the truncation errors and uniform upper bounds. We derive formulas for the derivatives and their errors as in [Fornæss and Gavosto 1992]. The main tool is to consider the power series expansions of the parametrization of the manifolds. Then we compute the values of the truncated series, and using Cauchy formulas we can conclude that the values are in certain explicit ranges, obtained applying the following technical lemma. We state the lemma for the real case. In the complex case, it can be modified in an obvious way.

Lemma 4.1. Let $f$ be a complex valued analytic function in $B\left(0, r_{a}\right) \times B\left(0, r_{\tau}\right)$ and such that $\left[a_{\min }, a_{\max }\right] \times$ $\left[\tau_{\min }, \tau_{\max }\right] \subset B\left(0, r_{a}\right) \times B\left(0, r_{\tau}\right)$. Assume that $f$ restricted to real values takes real values. Also assume that $a \in\left[a_{1}, a_{2}\right] \subset\left[a_{\min }, a_{\max }\right]$ and $\tau \in\left[\tau_{1}, \tau_{2}\right] \subset$ $\left[\tau_{\min }, \tau_{\max }\right]$. Suppose we have computed the value of the first $N$ terms of the power series expansion of $f$ in $\tau$ at a middle point $\left(a_{0}, \tau_{0}\right)$ and we have an upper bound on the truncation error $E_{N}$, say $f\left(a_{0}, \tau_{0}\right)=$ $f_{N}\left(a_{0}, \tau_{0}\right)+E_{N}$ with $\left|E_{N}\right| \leq \varepsilon_{N}, a_{0}=\frac{1}{2}\left(a_{1}+a_{2}\right)$, and $\tau_{0}=\frac{1}{2}\left(\tau_{1}+\tau_{2}\right)$. Then, for $a \in\left[a_{1}, a_{2}\right]$ and $\tau \in\left[\tau_{1}, \tau_{2}\right]$, we have

$$
\left|f(a, \tau)-f_{N}\left(a_{0}, \tau_{0}\right)\right|<R,
$$

where

$$
\begin{aligned}
& R=\frac{1}{2} \max (f)\left(\delta_{\tau}+\frac{1}{2}\left(\tau_{2}-\tau_{1}\right)\right)\left(\delta_{a}+\frac{1}{2}\left(a_{2}-a_{1}\right)\right) \\
& \quad \times\left(\frac{a_{2}-a_{1}}{\delta_{\tau} \delta_{a}^{2}}+\frac{\tau_{2}-\tau_{1}}{\delta_{\tau}^{2} \delta_{a}}\right)+\varepsilon_{N}, \\
& \delta_{a}=\min \left(\left|a_{\min }-a_{1}\right|,\left|a_{\max }-a_{2}\right|\right), \\
& \delta_{\tau}=\min \left(\left|\tau_{\min }-\tau_{1}\right|,\left|\tau_{\max }-\tau_{2}\right|\right) .
\end{aligned}
$$

Proof. The mean value theorem gives

$f(a, \tau)=f\left(a_{0}, \tau_{0}\right)+\frac{\partial f}{\partial a}\left(\xi_{a}, \xi_{\tau}\right)\left(a-a_{0}\right)$

$$
+\frac{\partial f}{\partial \tau}\left(\xi_{a}, \xi_{\tau}\right)\left(\tau-\tau_{0}\right)
$$

where $a \in\left[a_{1}, a_{2}\right]$ and $\tau \in\left[\tau_{1}, \tau_{2}\right]$. Using the Cauchy integral formula, we have

$$
f(a, \tau)=\frac{1}{(2 \pi i)^{2}} \int_{C_{A}} \int_{C_{\tau}} \frac{f\left(z_{1}, z_{2}\right)}{\left(z_{1}-a\right)\left(z_{2}-\tau\right)} d z_{1} d z_{2},
$$

where

$$
\begin{aligned}
C_{A} & =\left\{z_{1}:\left|z_{1}-a_{0}\right|=\delta_{a}+.5\left(a_{2}-a_{1}\right)\right\} \\
C_{\tau} & =\left\{z_{2}:\left|z_{2}-\tau_{0}\right|=\delta_{\tau}+.5\left(\tau_{2}-\tau_{2}\right)\right\} .
\end{aligned}
$$

Then

$$
\begin{aligned}
& \frac{\partial f(a, \tau)}{\partial a}=\frac{-1}{(2 \pi i)^{2}} \int_{C_{A}} \int_{C_{\tau}} \frac{f\left(z_{1}, z_{2}\right)}{\left(z_{1}-a\right)^{2}\left(z_{2}-\tau\right)} d z_{1} d z_{2}, \\
& \frac{\partial f(a, \tau)}{\partial \tau}=\frac{-1}{(2 \pi i)^{2}} \int_{C_{A}} \int_{C_{\tau}} \frac{f\left(z_{1}, z_{2}\right)}{\left(z_{1}-a\right)\left(z_{2}-\tau\right)^{2}} d z_{1} d z_{2} ;
\end{aligned}
$$

hence,

$$
\begin{aligned}
\left|\frac{\partial f(a, \tau)}{\partial a}\right| & \leq \frac{\max f}{(2 \pi)^{2}} \int_{C_{A}} \int_{C_{\tau}} \frac{1}{\left|z_{1}-a\right|^{2}\left|z_{2}-\tau\right|} d z_{1} d z_{2} \\
& \leq \max f \frac{\left(\delta_{\tau}+\frac{1}{2}\left(\tau_{2}-\tau_{1}\right)\right)\left(\delta_{a}+\frac{1}{2}\left(a_{2}-a_{1}\right)\right)}{\delta_{\tau} \delta_{a}^{2}} \\
\left|\frac{\partial f(a, \tau)}{\partial \tau}\right| & \leq \frac{\max f}{(2 \pi)^{2}} \int_{C_{A}} \int_{C_{\tau}} \frac{1}{\left|z_{1}-a\right|\left|z_{2}-\tau\right|^{2}} d z_{1} d z_{2} \\
& \leq \max f \frac{\left(\delta_{\tau}+\frac{1}{2}\left(\tau_{2}-\tau_{1}\right)\right)\left(\delta_{a}+\frac{1}{2}\left(a_{2}-a_{1}\right)\right)}{\delta_{\tau}^{2} \delta_{a}}
\end{aligned}
$$

Setting

$$
R_{1}=\frac{\partial f}{\partial a}\left(\xi_{a}, \xi_{\tau}\right)\left(a-a_{0}\right)+\frac{\partial f}{\partial \tau}\left(\xi_{a}, \xi_{\tau}\right)\left(\tau-\tau_{0}\right),
$$

we have

$$
\begin{aligned}
\left|R_{1}\right| \leq \frac{1}{2} \max f\left(\delta_{\tau}+\frac{1}{2}\left(\tau_{2}-\right.\right. & \left.\left.\tau_{1}\right)\right)\left(\delta_{a}+\frac{1}{2}\left(a_{2}-a_{1}\right)\right) \\
\times & \left(\frac{a_{2}-a_{1}}{\delta_{\tau} \delta_{a}^{2}}+\frac{\tau_{2}-\tau_{1}}{\delta_{\tau}^{2} \delta_{a}}\right),
\end{aligned}
$$

from which the lemma follows.

For example, we use this lemma in the following way. Recall from (2-1) that

$$
X^{u}(\tau, a)=\sum_{n=0}^{N} U_{n}(a) \tau^{n}+E(a, \tau, N) .
$$

Now suppose that we want to compute the value of $X^{u}(\tau, a)$ for $(\tau, a)$ in a neighborhood of $\left(\tau^{*}, a^{*}\right)$. We can compute $X_{N}^{u}\left(\tau^{*}, a^{*}\right)$ with $N$ found as described in Section 2. We can also obtain an upper bound for $E_{n}$ based on (2-2) Then we can apply the lemma with $f(a, \tau)=X^{u}(\tau, a)$ and we can estimate of the 
error that we make by approximating the value of $X^{u}(\tau, a)$ by $X_{N}^{u}\left(\tau^{*}, a^{*}\right)$ in a neighborhood of $\left(a^{*}, \tau^{*}\right)$. A systematic application of this lemma gives us a way of checking all the conditions for the existence of the generic homoclinic tangency. See [Fornæss and Gavosto 1992] for details in checking the conditions. If any of the conditions fails, we go back to Step $\mathrm{D}$ to obtain a closer approximation as long as we are working with the right precision. If we need more digits of precision, for example, to compute $X_{N}^{u}\left(\tau^{*}, a^{*}\right)$, we go back to Step C.

\section{APPLICATIONS AND COMPUTATIONAL DIFFICULTIES}

To illustrate the type of numerical computational difficulties involved, we have displayed in Table 1 the approximate size of the coefficients of the unstable manifold for some values of the parameter. The size of these coefficients goes to zero since the unstable manifold is entire. Recall that its parametrization is $\left(\sum_{n=0}^{\infty} U_{n}(a) \tau^{n}, \sum_{n=0}^{\infty} U_{n}(a)\left(\tau /\left(\lambda^{u}\right)\right)^{n}\right)$ for all values of $\tau \in \mathbb{C}$. Nevertheless, the computations for $a$ around 0.36 to 0.50 do need many digits of precision since the size of $U_{n}$ starts to decrease only for very large values of $n$. For $a=0.36, U_{500}$ is still of the order of $10^{647}$. Using only double precision in the computations, we have observed graphically tangencies for $a$ in the interval $[0.36,0.50]$ that do not exist when higher precision is used.

In [Kan et al. 1995], some numerical evidence and heuristics arguments are presented for the validity of the following conjecture.

Conjecture 5.1 [Kan et al. 1995]. The real Hénon mappings have the Newhouse intervals

$$
\begin{aligned}
& I_{1}=[1.2702,12997], \\
& I_{2}=[1.3087,13233], \\
& I_{3}=[1.3238,14200] .
\end{aligned}
$$

A Newhouse interval for the family $H_{a}$ is an interval containing a dense subset $J$ so that, for any $a \in J$, $H_{a}$ has a generic homoclinic tangency.

Using our algorithm, we can show the following.

Theorem 5.2. The Hénon Map has a generic homoclinic tangency for $a=1.305045 \ldots$.

This shows that there are Newhouse intervals for the Hénon map other than the ones in Conjecture 5.1.

\begin{tabular}{|ccrrrr|}
\hline \multicolumn{1}{c}{$\log$} & \multicolumn{1}{c}{$\log$} & $\log$ & $\log$ \\
$a$ & $U_{0}$ & $-U_{10}$ & \multicolumn{1}{c}{$U_{100}$} & \multicolumn{1}{c|}{$U_{200}$} & $\left|U_{500}\right|$ \\
\hline 0.36 & 0.95 & 10.31 & 8.14 & 738.19 & $\star 1490.57$ \\
0.38 & 0.94 & 8.06 & 110.04 & 221.29 & 550.66 \\
0.40 & 0.93 & 4.05 & 55.49 & 107.52 & 251.55 \\
0.42 & 0.92 & 1.96 & 24.64 & 41.51 & 72.67 \\
0.44 & 0.90 & 0.50 & 1.68 & -8.57 & -65.79 \\
0.46 & 0.89 & -0.63 & -17.26 & -50.39 & -183.29 \\
0.48 & 0.88 & -1.61 & -33.72 & -87.17 & -287.67 \\
0.50 & 0.87 & -2.41 & -48.48 & -120.32 & -382.86 \\
0.55 & 0.85 & -4.07 & -80.49 & -193.05 & -593.67 \\
0.60 & 0.83 & -5.52 & -107.89 & -256.05 & -778.25 \\
0.80 & 0.76 & -9.43 & -193.68 & -456.12 & -1371.66 \\
0.92 & 0.72 & -11.51 & -234.48 & -552.09 & -1659.06 \\
1.03 & 0.70 & -12.46 & -267.40 & -629.69 & -1892.31 \\
1.10 & 0.68 & -13.24 & -286.57 & -674.93 & -2028.57 \\
1.30 & 0.64 & -15.19 & -335.31 & -790.17 & -2376.09 \\
1.40 & 0.63 & -16.05 & -356.94 & -841.44 & -2530.70 \\
1.50 & 0.61 & -16.83 & -377.07 & -889.23 & -2674.85 \\
\hline
\end{tabular}

TABLE 1. Approximate coefficients of the unstable manifold for selected values of $a$. In the rightmost column, the $\star$ indicates the only entry for which $U_{500}$ is negative.

Note that this tangency is located for a parameter value where the Hénon mapping appears to have a discrete attractor (see Figure 2). The main future application that we have for this method is to locate Fatou Bieberbach domains in $\mathbb{C}^{2}$. We have some preliminary results, to appear elsewhere, based on our precise locations of generic homoclinic tangencies.

\section{ACKNOWLEDGEMENTS}

We thank the referee for comments and suggestions that improved our manuscript.

\section{REFERENCES}

[Benedicks and Carleson 1991] M. Benedicks and L. Carleson, "The dynamics of the Hénon map", Ann. of Math. (2) 133:1 (1991), 73-169.

[Buzzard 1997] G. T. Buzzard, "Infinitely many periodic attractors for holomorphic maps of 2 variables", Ann. of Math. (2) 145:2 (1997), 389-417.

[Fornæss and Gavosto 1991] J. E. Fornæss and E. A. Gavosto, "Existence d'attracteurs étranges pour certaines applications de Hénon", C. R. Acad. Sci. Paris Sér. I Math. 313:8 (1991), 495-498. 


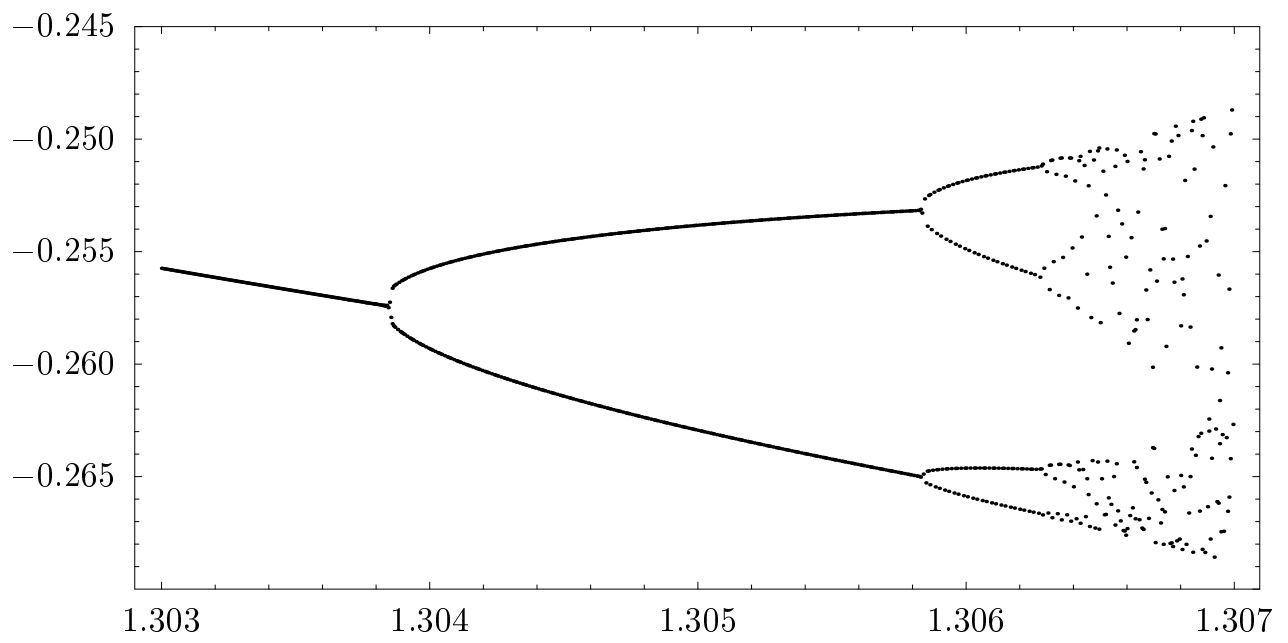

FIGURE 2. Bifurcation diagram of the real Hénon family for $a \in(1.303,1.307)$. The tangency is located at $(1.305,-0.388)$, about $25 \mathrm{~cm}$ (10 inches) below the lower edge of the graph.

[Fornæss and Gavosto 1992] J. E. Fornæss and E. A. Gavosto, "Existence of generic homoclinic tangencies for Hénon mappings", J. Geom. Anal. 2:5 (1992), 429444 .

[Franceschini and Russo 1981] V. Franceschini and L. Russo, "Stable and unstable manifolds of the Hénon mapping", J. Statist. Phys. 25:4 (1981), 757-769.

[Friedland and Milnor 1989] S. Friedland and J. Milnor, "Dynamical properties of plane polynomial automorphisms", Ergodic Theory Dynamical Systems 9:1 (1989), 67-99.

[Gavosto $\geq 1999]$ E. Gavosto, "Attracting basins in $\mathbb{P}^{2}$ ". To appear in J. Geom. Anal.

[Hénon 1976] M. Hénon, "A two-dimensional mapping with a strange attractor", Comm. Math. Phys. 50:1 (1976), 69-77.
[Kan et al. 1995] I. Kan, H. Koçak, and J. A. Yorke, "Persistent homoclinic tangencies in the Hénon family", Phys. D 83:4 (1995), 313-325.

[Mora and Viana 1993] L. Mora and M. Viana, "Abundance of strange attractors", Acta Math. 171:1 (1993), 1-71.

[Newhouse 1974] S. E. Newhouse, "Diffeomorphisms with infinitely many sinks", Topology 13 (1974), 918.

[Robinson 1983] C. Robinson, "Bifurcation to infinitely many sinks", Comm. Math. Phys. 90:3 (1983), 433459 .

[Stens $\varnothing$ nes 1997] B. Stensønes, "Fatou-Bieberbach domains with $C^{\infty}$-smooth boundary", Ann. of Math. (2) 145:2 (1997), 365-377.

John Erik Fornæss, Mathematics Department, University of Michigan, Ann Arbor, MI 48109, United States (fornaess@umich.edu)

Estela A. Gavosto, Department of Mathematics, University of Kansas, Lawrence, KS 66049, United States (gavosto@math.ukans.edu)

Received December 11, 1997; accepted in revised from August 24, 1998 